Supplement of

\title{
An Estimate of Global, Regional and Seasonal Cirrus Cloud Radiative Effects Contributed by Homogeneous Ice Nucleation
}

David L. Mitchell et al.

Correspondence to: David Mitchell (david.mitchell@dri.edu) 
The following subroutine in Fortran90 was used in the CALIPSO simulation to determine $\mathrm{D}_{\mathrm{e}}$ (retrieved median effective diameter) for a given

temperature, longitude, season and land-fraction. Look-up-tables (LUTs) for $D_{e}$ are embedded in the subroutine, specified by latitude and season. The $D_{e}$ entries in the LUTs are separated by a temperature of $4 \mathrm{~K}$, with the minimum and maximum LUT temperature being 168 and 268K (giving $25 \mathrm{De}_{\mathrm{e}}$ entries). After a temperature supplied by the main program is related to a given LUT (defined by season and latitude range), De over ocean and land are determined by interpolation between the two nearest indexed $D_{e}$ values in the respective LUT. Once $D_{e}$ for ocean and land are calculated (deo \& del), $D_{e}$ is calculated as a function of land-fraction, given by the variable luindex. Finally, when $D_{e}$ lies within \pm 5 degrees of a latitude boundary (used to define $\mathrm{D}_{\mathrm{e}}$ categories), then $\mathrm{D}_{\mathrm{e}}$ is calculated from a line equation that is defined by two latitudes \pm 5 degrees latitude from the latitude being solved for. This prevents abrupt transitions in $D_{e}$ from occurring between $D_{e}$-latitude categories.

The code below should be mostly self-explanatory upon inspection, given the above explanation. The temperature variable = tamb. LUTs are labeled by latitude zone, whether the zone is in the northern or southern hemisphere, whether the LUT is for land or ocean De values, and the season it corresponds to. For example, detab6090Nldjf indicates $\mathrm{D}_{\mathrm{e}}$ table for 60 to 90 deg. N. latitude, land De values, and December-January-February. Similarly, detab6090Nojja indicates De table for 60 to 90 deg. N. latitude, ocean $D_{e}$ values, and June-July-August. The $D_{e}$ LUT values are in microns. The subroutine code is as follows:

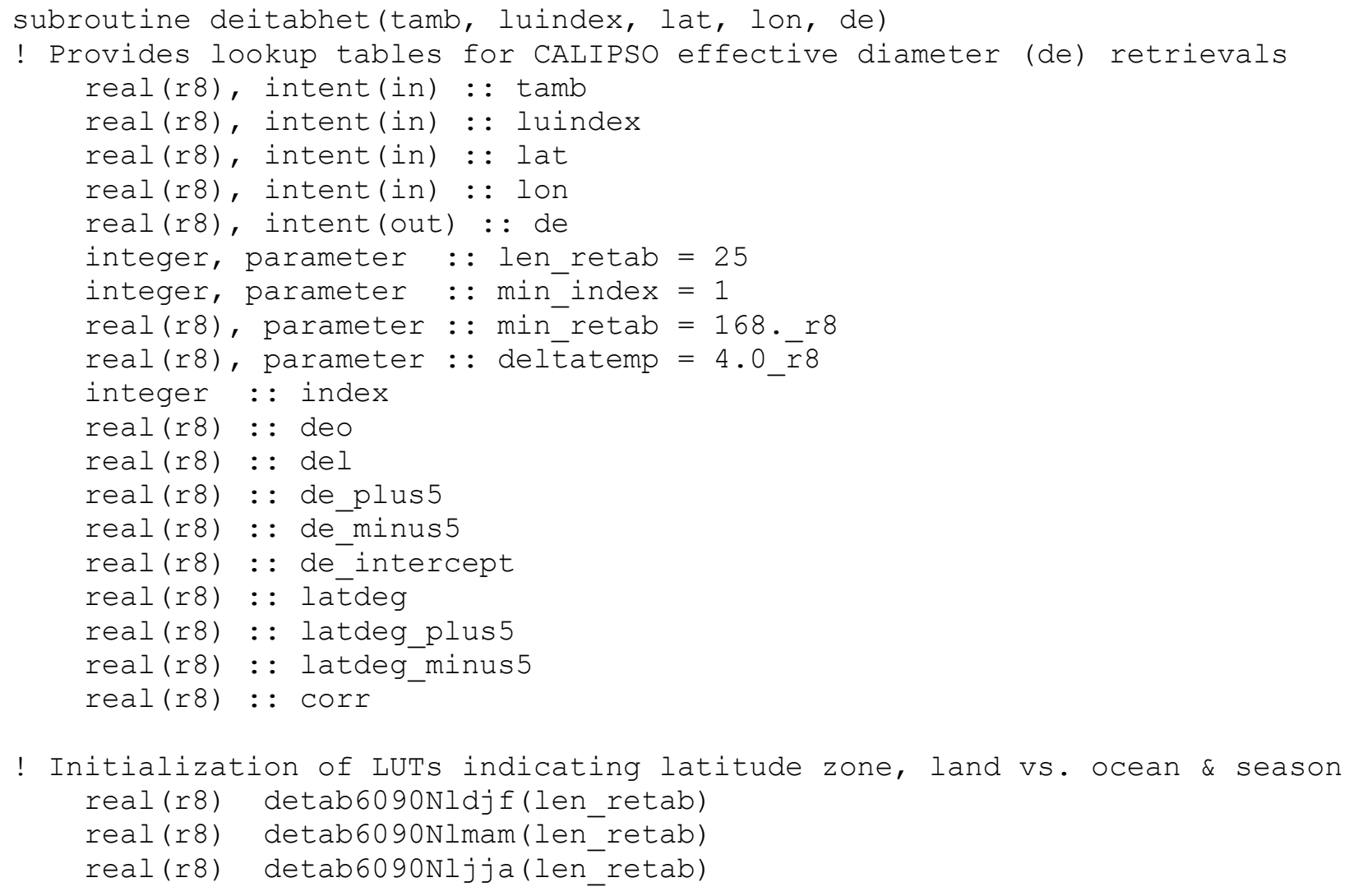

! Initialization of LUTs indicating latitude zone, land vs. ocean \& season real(r8) detab6090Nldjf(len_retab)

real(r8) detab6090Nlmam(len_retab)

real(r8) detab6090Nljja(len_retab) 


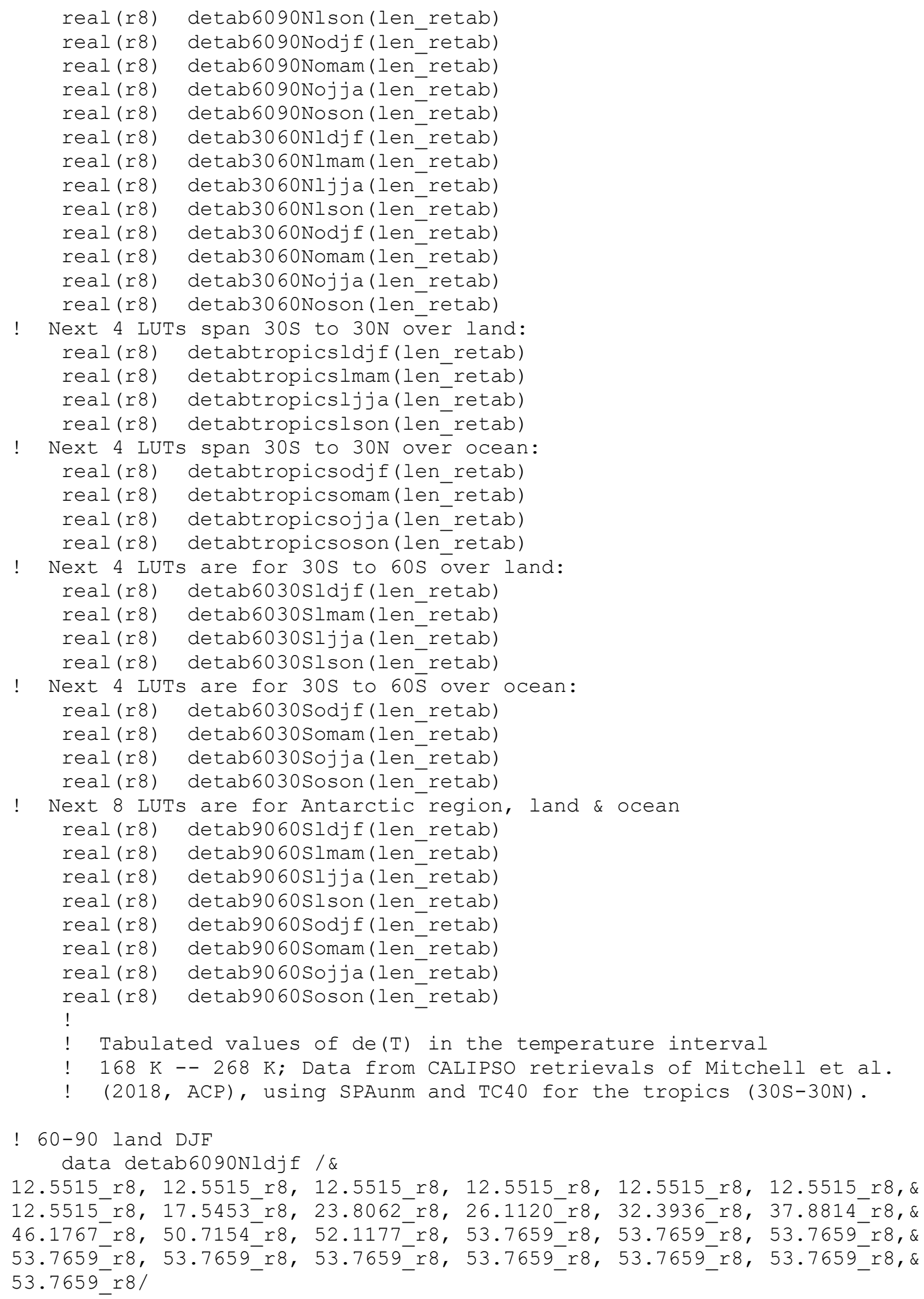


save detab6090Nldjf

! 60-90 land MAM

data detab6090Nlmam / \&

$31.3916 r 8,31.3916 r 8,31.3916 r 8,31.3916 r 8,31.3916 r 8,31.3916 \_r 8, \&$ $31.3916 r 8,31.3916 r 8,31.3916 r 8,31.3916 r 8,29.8408$ r8, 35.5569_r8, \& $42.8987 r 8,48.5698 r 8,51.8230 r 8,51.8230 r 8,51.8230 r 8,51.8230 r 8, \&$ 51.8230_r8, 51.8230_r8, 51.8230_r8, 51.8230_r8, 51.8230_r8, 51.8230_r8, \& $51.8230-r 8 /$

save detab6090Nlmam

! 60-90 land JJA

data detab6090Nljja / \&

$35.4264 r 8,35.4264 r 8,35.4264 r 8,35.4264 r 8,35.4264 r 8,35.4264 r 8, \&$ $35.4264 r 8,35.4264 r 8,35.4264 r 8,35.4264 r 8,35.4264 r 8,45.1069 r 8, \&$ 50.5470_r8, 54.5277_r8, 56.2471_r8, 56.2471_r8, 56.2471_r8, 56.2471_r8,\& 56.2471_r8, 56.2471_r8, 56.2471_r8, 56.2471_r8, 56.2471_r8, 56.2471_r8,\& 56.2471 r $8 /$

save detab6090Nljja

! 60-90 land SON

data detab6090Nlson /\&

15.1583 r8, 15.1583r8, 15.1583r8, 15.1583r8, 15.1583r8, 15.1583_r8, \& 15.1583 r8, 15.1583 r8, 15.1583 r8, 15.1583_r8, 29.7252_r8, 34.3148_r8, \& $38.9208 r 8,47.8403^{-} r 8,48.7599 r 8,53.5761 r 8,53.5761 r 8,53.5761 r 8, \&$ 53.5761_r8, 53.5761_r8, 53.5761_r8, 53.5761_r8, 53.5761_r8, 53.5761_r8,\& 53.5761 r8/

save detab6090Nlson

! 60-90 ocean DJF data detab6090Nodjf /\&

24.5915 r8, 24.5915_r8, 24.5915_r8, 24.5915_r8, 24.5915_r8, 24.5915_r8,\& $24.5915^{-} r 8,24.5915^{-} r 8,32.8914^{-} r 8,33.5891^{-} \mathrm{r} 8,38.8793^{-} \mathrm{r} 8,41.5984 \mathrm{r} 8, \&$ 49.5036_r8, 56.6347_r8, 58.1320_r8, 58.1320_r8, 58.1320_r8, 58.1320_r8,\& 58.1320_r8, 58.1320_r8, 58.1320_r8, 58.1320_r8, 58.1320_r8, 58.1320_r8,\& $58.1320-r 8 /$

save detab6090Nodjf

! 60-90 ocean MAM

data detab6090Nomam /\&

23.0161_r8, 23.0161_r8, 23.0161_r8, 23.0161_r8, 23.0161_r8, 23.0161_r8,\& 23.0161_r8, 23.0161_r8, 23.0161_r8, 23.0161_r8, 25.9148_r8, 43.3420_r8, \& 48.9045_r8, 54.4704_r8, 58.1393_r8, 70.3760_r8, 70.3760_r8, 70.3760_r8, \& 70.3760_r8, 70.3760_r8, 70.3760_r8, 70.3760_r8, 70.3760_r8, 70.3760_r8, \& $70.3760-r 8 /$

save detab6090Nomam

! 60-90 ocean JJA

data detab6090Nojja /\&

$25.0766 r 8,25.0766 r 8,25.0766 r 8,25.0766 r 8,25.0766 \_r 8,25.0766 \_r 8, \&$ 25.0766_r8, 25.0766_r8, 25.0766_r8, 25.0766_r8, 25.0766_r8, 25.0766_r8,\& 40.0769 r8, 52.0571_r8, 56.8702_r8, 56.8702_r8, 56.8702_r8, 56.8702_r8,\& $56.8702 \_r 8,56.8702 \_r 8,56.8702 \_r 8,56.8702 \_r 8,56.8702 \_r 8,56.8702 \_r 8, \&$ $56.8702-r 8 /$ 
save detab6090Nojja

! 60-90 ocean SON

data detab6090Noson /\&

17.5430_r8, 17.5430_8, 17.5430_r8, 17.5430_r8, 17.5430_r8, 17.5430_r8,\& 17.5430_r8, 17.5430_r8, 17.5430_r8, 28.0634_r8, 34.9437_r8, 40.9240_r8,\& $45.5826 r 8,52.0371^{-} r 8,55.6067 r 8,55.6067 r 8,55.6067 r 8,55.6067 r 8, \&$ 55.6067_r8, 55.6067_r8, 55.6067_r8, 55.6067_r8, 55.6067_r8, 55.6067_r8,\& $55.6067-r 8 /$

save detab6090Noson

! 30-60 land DJF

data detab3060Nldjf /\&

$13.7516 r 8,13.7516 r 8,13.7516 r 8,13.7516 r 8,13.7516 r 8,13.7516 r 8, \&$ $13.7516 r 8,13.7516 r 8,24.7085 r 8,26.9770 r 8,34.6243 r 8,42.6144 r 8, \&$ 48.3151_r8, 53.9897_8, 55.4510r8, 55.6016r8, 55.6016r8, 55.6016r8,\& 55.6016_r8, 55.6016_r8, 55.6016_r8, 55.6016_r8, 55.6016_r8, 55.6016_r8,\& $55.6016 r 8 /$

save detab3060Nldjf

\section{! 30-60 land MAM}

data detab3060Nlmam /\&

$22.4826 r 8,22.4826 r 8,22.4826 r 8,22.4826 r 8,22.4826 r 8,22.4826 r 8, \&$ $22.4826 \_r 8,22.4826^{-} r 8,22.4826^{-} r 8,30.5000^{-} r 8,41.7793$ r8 $, 49.3616^{-} r 8, \&$ $54.9672 r 8,60.5807 r 8,61.9360 r 8,61.9360 r 8,61.9360 r 8,61.9360^{-} r 8, \&$ 61.9360_r8, 61.9360_r8, 61.9360_r8, 61.9360_r8, 61.9360_r8, 61.9360_r8,\& 61.9360 r8/

save detab3060Nlmam

! 30-60 land JJA data detab3060Nljja / \&

$27.9527 \_r 8,27.9527 \_r 8,27.9527 \_r 8,27.9527$ r8, 27.9527_r8, 27.9527_r8,\& $27.9527^{-} r 8,27.9527^{-} r 8,39.8649^{-} r 8,47.3965^{-} r 8,51.3956^{-} r 8,52.4833^{-} r 8, \&$ 56.2833_r8, 62.9856_r8, 66.0834_r8, 67.4987_r8, 83.3788_r8, 83.3788_r8, \& 83.3788_r8, 83.3788_r8, 83.3788_r8, 83.3788_r8, 83.3788_r8, 83.3788_r8, \& 83.3788 r8/

save detab3060Nljja

! 30-60 land SON data detab3060Nlson /\&

$23.9838 \_r 8,23.9838 \_r 8,23.9838 \_r 8,23.9838 \_r 8,23.9838 \_r 8,23.9838 \_r 8, \&$ $23.9838^{-} r 8,23.9838^{-} r 8,23.9838^{-} r 8,24.5728^{-} r 8,33.9347^{-} r 8,42.8478 r 8, \&$ 51.3945_r8, 56.6141_r8, 59.9297_r8, 59.9297_r8, 59.9297_r8, 59.9297_r8,\& 59.9297_r8, 59.9297_r8, 59.9297_r8, 59.9297_r8, 59.9297_r8, 59.9297_r8,\& 59.9297 r8/

save detab3060Nlson

! 30-60 ocean DJF data detab3060Nodjf /\&

23.0164_r8, 23.0164_r8, 23.0164_r8, 23.0164_r8, 23.0164_r8, 23.0164_r8, \& 23.0164_r8, 23.0164_r8, 28.7222_r8, 35.0160_r8, 42.4562_r8, 48.0642_r8,\& 53.1924_r8, 57.9852_r8, 61.3784_r8, 61.3784_r8, 61.3784_r8, 61.3784_r8, \& 61.3784_r8, 61.3784_r8, 61.3784_r8, 61.3784_r8, 61.3784_r8, 61.3784_r8, \& 61.3784 r8/ 
save detab3060Nodjf

! 30-60 ocean MAM

data detab3060Nomam /\&

$25.2382 \_8,25.2382 \_8,25.2382 \_8,25.2382 r 8,25.2382 \_8,25.2382 \_r 8, \&$ 25.2382_r8, 25.2382_r8, 25.2382_r8, 37.0475_r8, 42.2693_r8, 49.3426_r8,\& 55.5881_r8, 61.8791_r8, 62.8743_r8, 69.7545_r8, 69.7545_r8, 69.7545_r8,\& 69.7545_r8, 69.7545_r8, 69.7545_r8, 69.7545_r8, 69.7545_r8, 69.7545_r8,\& 69.7545 r8/

save detab3060Nomam

! 30-60 ocean JJA

data detab3060Nojja / \&

$31.5030 r 8,31.5030 r 8,31.5030 r 8,31.5030 r 8,31.5030 r 8,31.5030 r 8, \&$ $31.5030^{-} r 8,31.5030^{-} r 8,31.5030^{-} r 8,48.4480^{-} r 8,53.0315 r 8,54.8413^{-} r 8, \&$ 57.3852_r8, 62.4347_r8, 66.9753_r8, 66.6357_r8, 66.6357_r8, 66.6357_r8,\& 66.6357_r8, 66.6357_r8, 66.6357_r8, 66.6357_r8, 66.6357_r8, 66.6357_r8,\& 66.6357 r8/

save detab3060Nojja

! 30-60 ocean SON

data detab3060Noson / \&

$25.3306 \_r 8,25.3306 \_r 8,25.3306 \_r 8,25.3306 \_r 8,25.3306 \_r 8,25.3306 \_r 8, \&$ 25.3306_r8, 25.3306_r8, 35.4344_r8, 39.0768_r8, 47.1180_r8, 52.0583_r8,\& $56.1453^{-} r 8,60.8797 r 8,61.6096 r 8,61.6096 r 8,61.6096 r 8,61.6096 r 8, \&$ 61.6096_r8, 61.6096_r8, 61.6096_r8, 61.6096_r8, 61.6096_r8, 61.6096_r8,\& $61.6096 r 8 /$

save detab3060Noson

! $-30+30$ land DJF

data detabtropicsldjf /\&

14.1112_r8, 14.1112_r8, 14.1112_r8, 14.1112_r8, 14.1112_r8, 14.1112_r8, \& 21.6484_r8, 26.4968_r8, 35.2015_r8, 44.1877_r8, 51.8588_r8, 59.4626_r8,\& $64.3535 r 8,72.8794 r 8,69.0298 r 8,69.0298 r 8,73.0000^{-} r 8,73.0000^{-} r 8, \&$ 73.0000_r8, 73.0000_r8, 73.0000_r8, 73.0000_r8, 73.0000_r8, 73.0000_r8, \& 73.0000 r8/

save detabtropicsldjf

! $-30+30$ land MAM

data detabtropicslmam /\&

11.2549_r8, 11.2549_r8, 11.2549_r8, 11.2549_r8, 11.2549_r8, 16.4684_r8, \& 20.9409_r8, 28.9171_r8, 37.0794_r8, 45.4198_r8, 51.5109_r8, 58.4374_r8,\& 66.7202_r8, 73.7770_r8, 92.2404_r8, 105.474_r8, 105.474_r8, 105.474_r8,\& 105.474_r8, 105.474_r8, 105.474_r8, 105.474_r8, 105.474_r8, 105.474_r8,\& $105.474-r 8 /$

save detabtropicslmam

! $-30+30$ land JJA

data detabtropicsljja /\&

14.8852_r8, 14.8852_r8, 14.8852_r8, 14.8852_r8, 14.8852_r8, 14.8852_r8, \& 18.4412r8, 23.8292r8, 34.5683r8, 40.6783r8, 50.2099r8, 57.4005 r8,\& 64.2896_r8, 76.3264_r8, 88.3262_r8, 105.707_r8, 105.707_r8, 105.707_r8,\& 105.707_r8, 105.707_r8, 105.707_r8, 105.707_r8, 105.707_r8, 105.707_r8,\& 


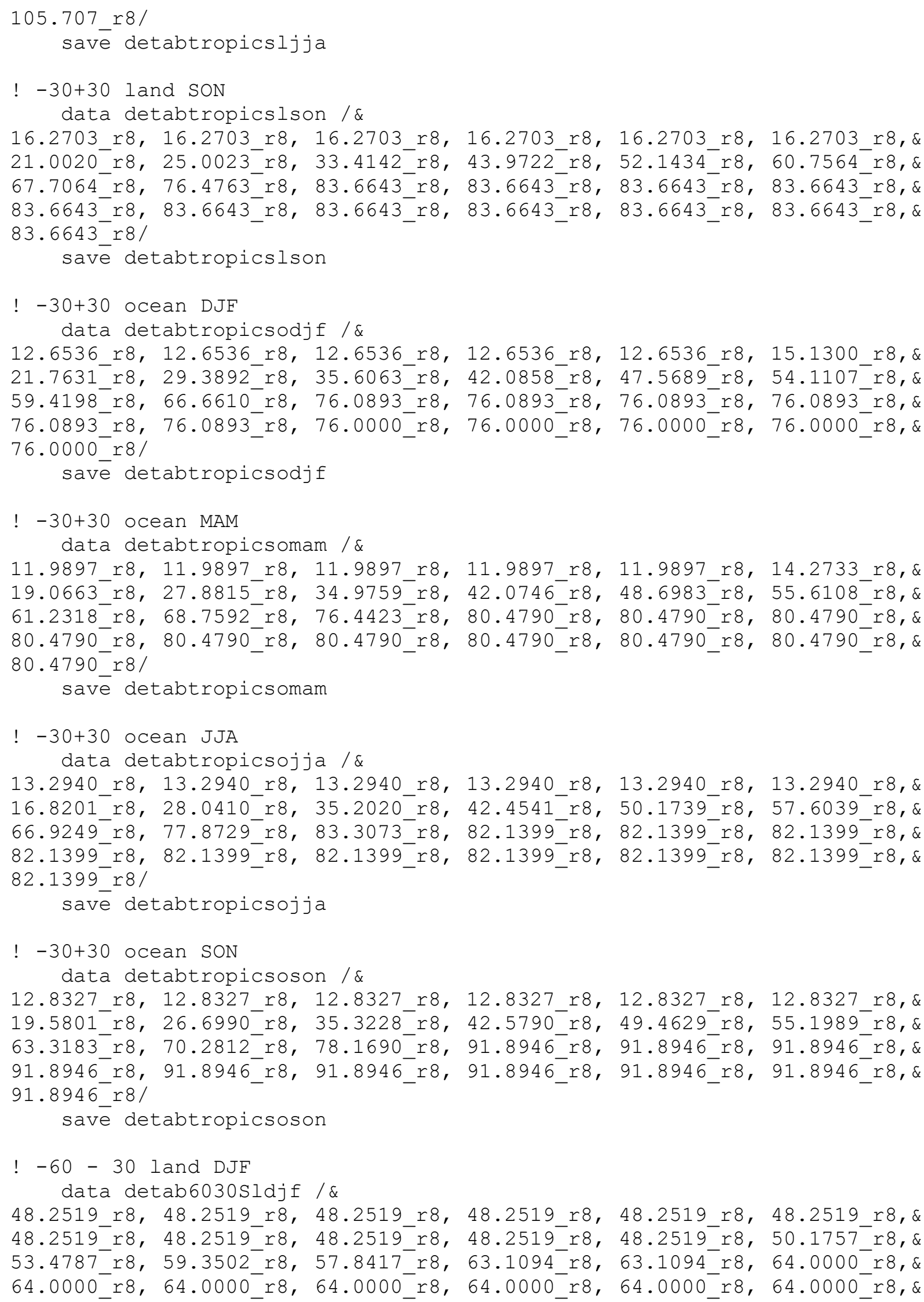




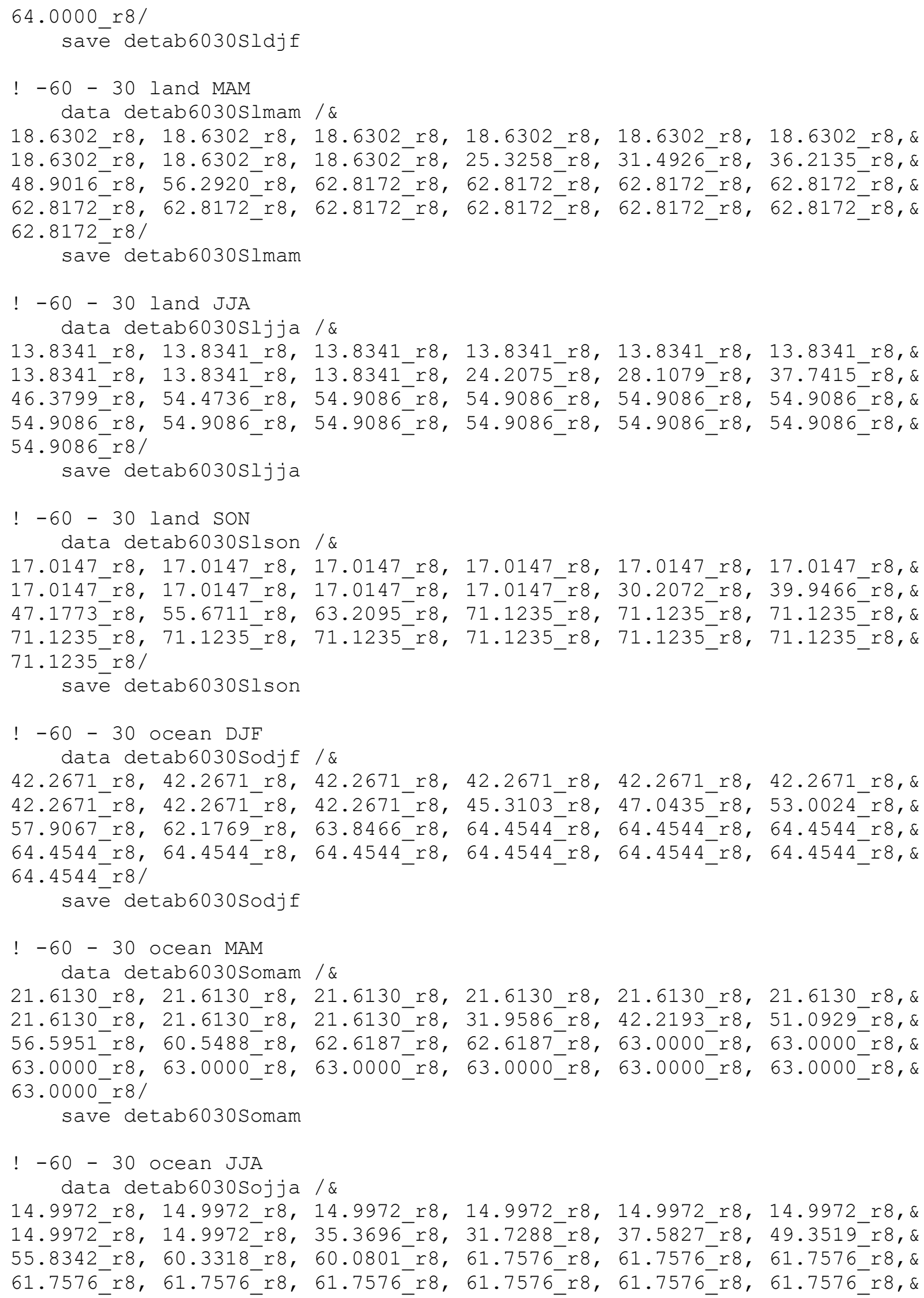




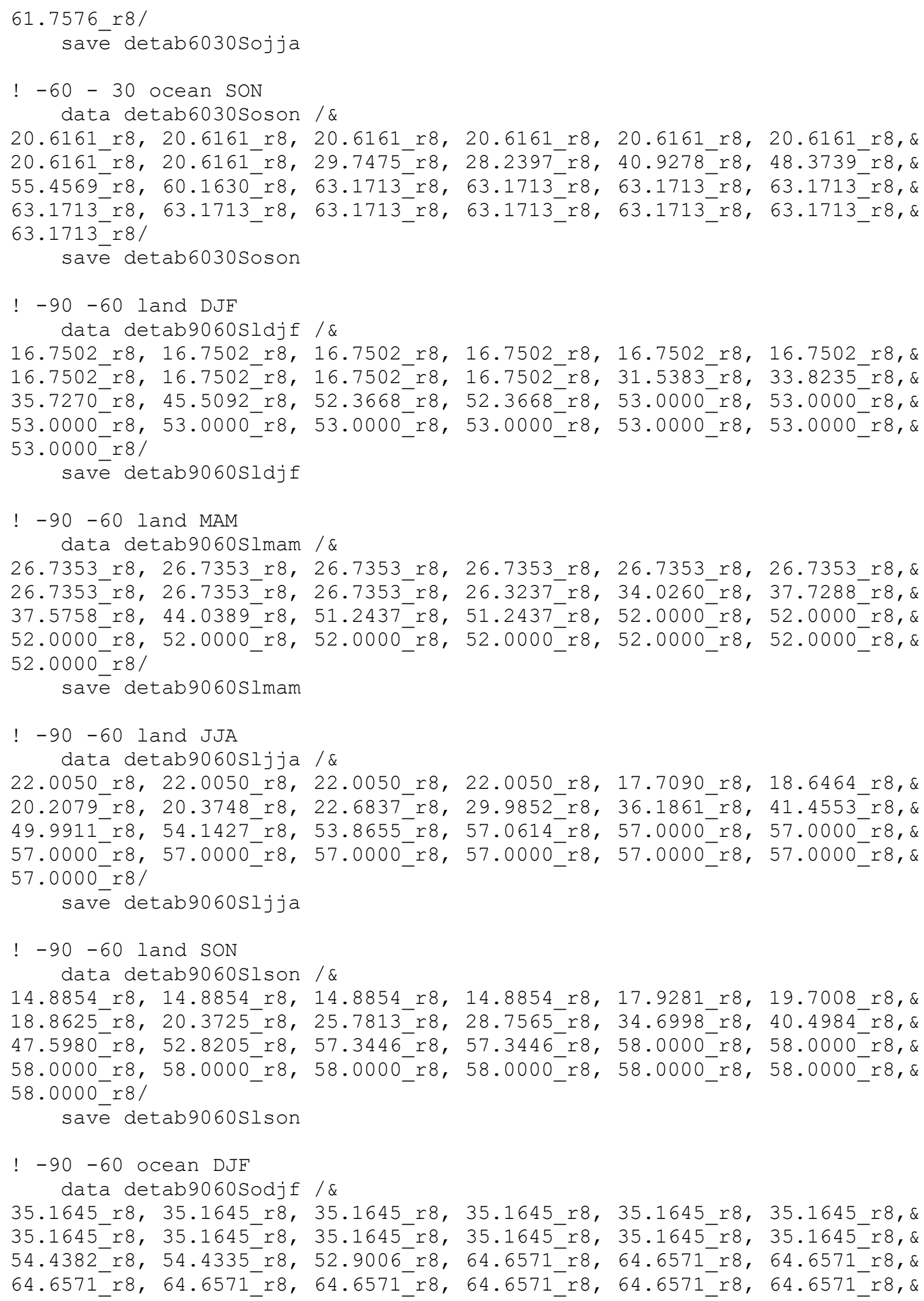




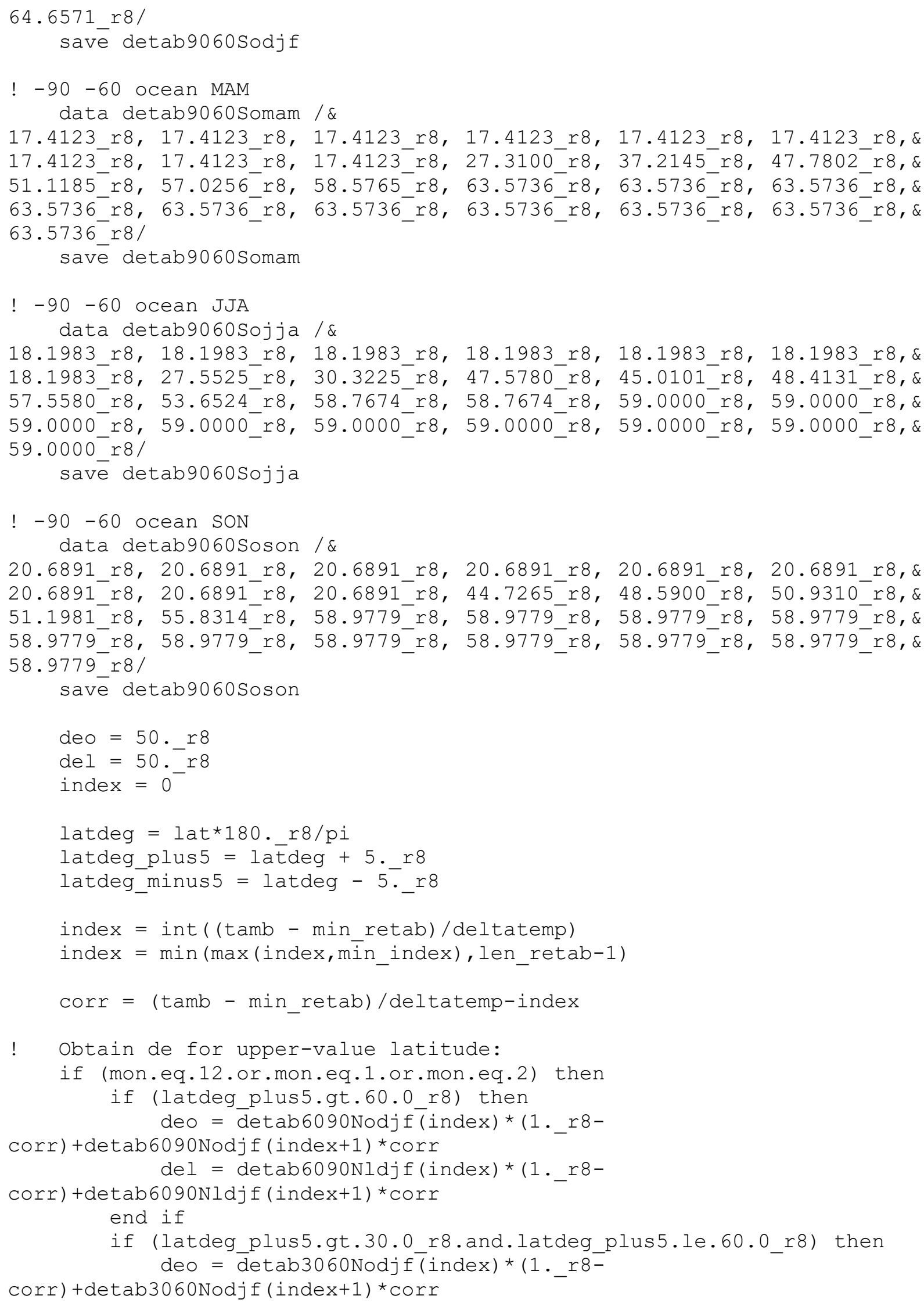


$\operatorname{del}=\operatorname{detab3060Nldjf(index)*(1..r8-}$

corr $)+\operatorname{detab3060Nldjf(index+1)*\operatorname {corr}}$

endif

if (latdeg_plus5.ge.-30.0_r8.and.latdeg_plus5.le.30.0_r8) then

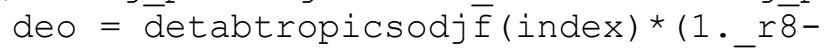

corr $)+$ detabtropicsodjf (index+1) ${ }^{*} \operatorname{corr}$

del $=$ detabtropicsldjf $($ index $) *(1 ., r 8-$

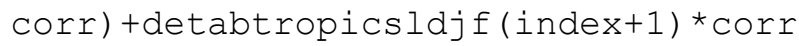

endif

if (latdeg_plus5.ge.-60.0_r8.and.latdeg_plus5.1t.-30.0_r8) then deo $=$ detab6030Sodjf $(\bar{i}$ dex $) *(1$. r8 -

corr $)+\operatorname{detab6030Sodjf~(index+1)*\operatorname {corr}}$

$\operatorname{del}=\operatorname{detab6030sldjf(index)*(1..r8-~}$

corr $)+\operatorname{detab6030sldjf(index+1)*\operatorname {corr}}$

endif

if (latdeg_plus5.1t.-60.0_r8) then

deo $=$ detab9060Sodjf $(\bar{i}$ dex $) *(1 . . r 8-$

corr $)+\operatorname{detab9060Sodjf~(index+1)*} \operatorname{corr}$

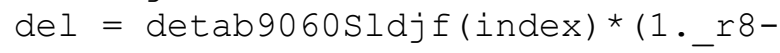

corr $)+\operatorname{detab9060Sldjf(index+1)~}{ }^{*} \operatorname{corr}$

end if

end if

if (mon.eq.3.or.mon.eq.4.or.mon.eq.5) then

if (latdeg_plus5.gt.60.0_r8) then

deo $=$ detab6090Nomam(index $) *(1$. r $8-$

corr $)+\operatorname{detab6090Nomam~(index+1)~}{ }^{*} \operatorname{corr}$

del $=\operatorname{detab6090Nlmam}($ index $) *(1 . r 8-$

corr) +detab6090Nlmam (index+1) * corr

end if

if (latdeg_plus5.gt.30.0_r8.and.latdeg_plus5.le.60.0_r8) then

deo $=$ detab3060Nomam (index)*(1._r8-

corr $)+\operatorname{detab3060Nomam~(index+1)*\operatorname {corr}}$

$\operatorname{del}=\operatorname{detab3060Nlmam}($ index $) *(1 . r$ r8-

corr $)+\operatorname{detab3060Nlmam}\left(\right.$ index+1) ${ }^{*} \operatorname{corr}$

endif

if (latdeg_plus5.ge.-30.0_r8.and.latdeg_plus5.1e.30.0_r8) then deo $=\overline{\text { detabtropicsomam }}($ index $) *(1, \ldots \overline{8}-$

corr $)+$ detabtropicsomam (index+1) * $\operatorname{corr}$

del = detabtropicslmam (index) $*\left(1 . \_r 8-\right.$

corr) +detabtropicslmam (index+1)* $\operatorname{corr}$

endif

if (latdeg_plus5.ge.-60.0_r8.and.latdeg_plus5.1t.-30.0_r8) then deo $=$ detab6030Somam (index)*(1._r8-

corr $)+\operatorname{detab6030Somam~(index+1)*\operatorname {corr}}$

del $=\operatorname{detab6030Slmam}($ index $) *(1 \cdot$ r $8-$

corr $)+\operatorname{detab6030SImam~(index+1)~}{ }^{*} \operatorname{corr}$

endif

if (latdeg_plus5.1t.-60.0_r8) then

deo $=$ detab9060Somam (index)*(1._r8-

corr) +detab9060 Somam (index+1) ${ }^{*} \operatorname{corr}$

del $=\operatorname{detab9060Slmam}($ index $) *(1$. r8 -

corr) +detab9060Slmam (index+1) * corr

end if

end if 


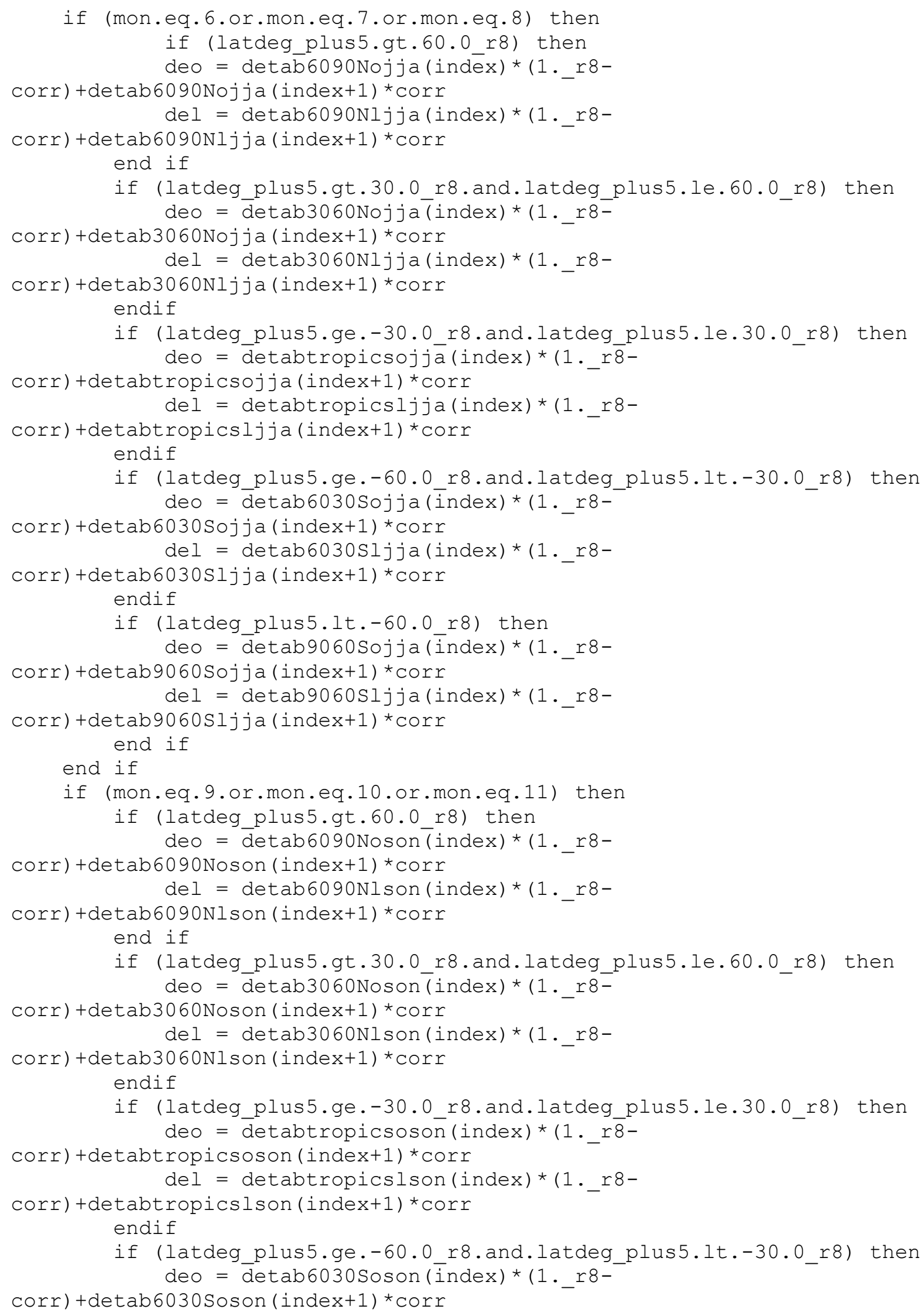




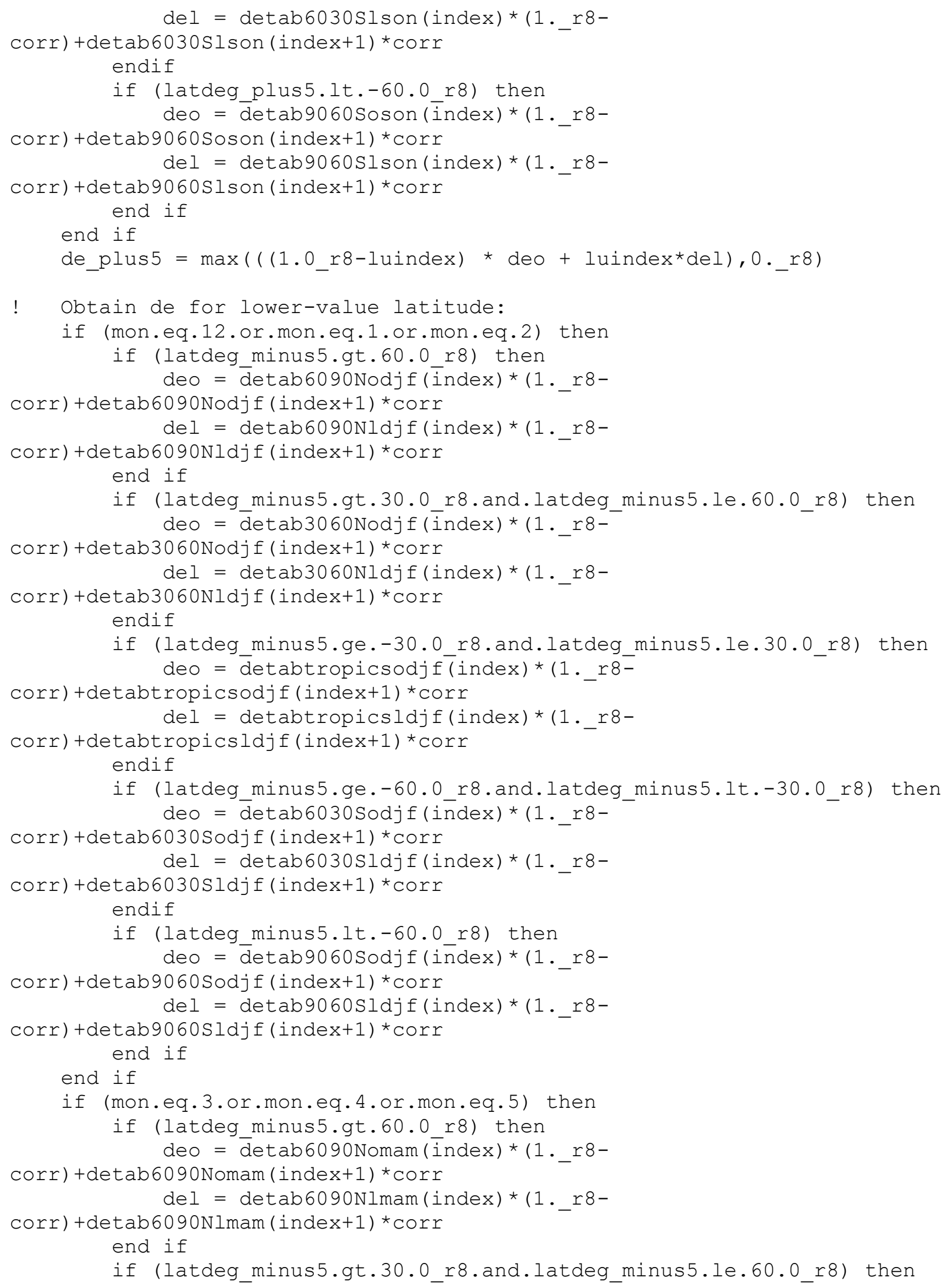




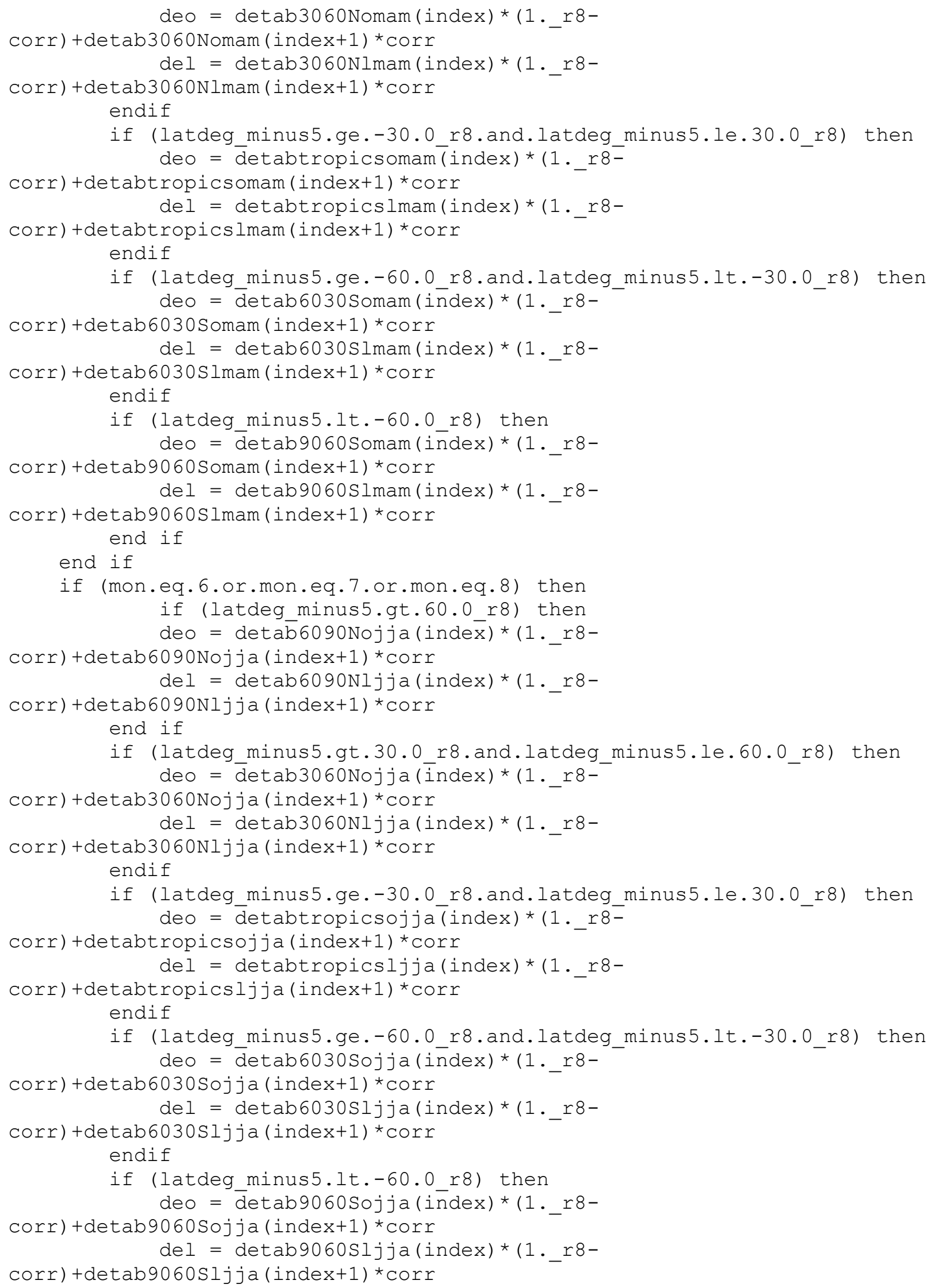




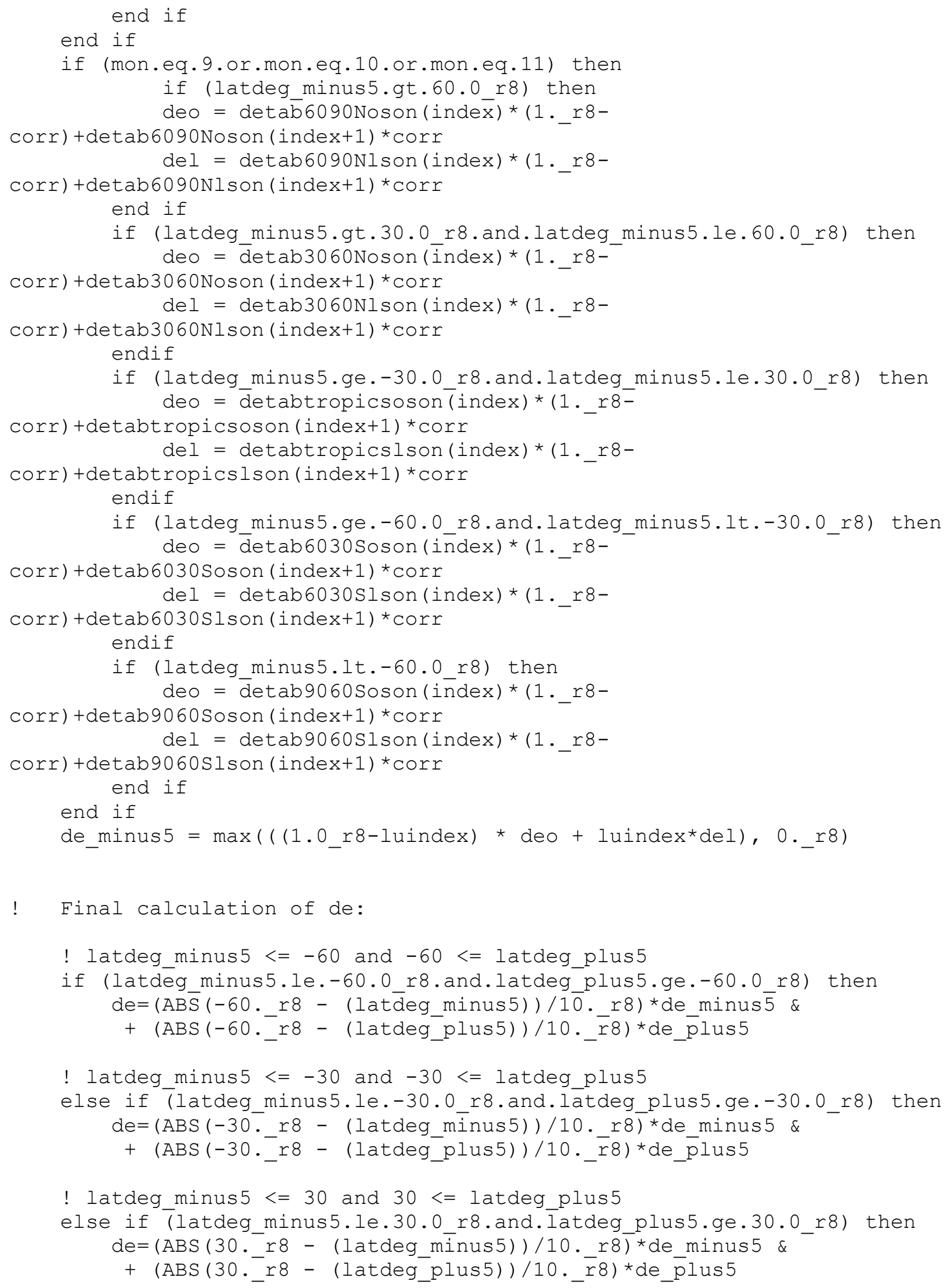


! latdeg_minus $5<=60$ and $60<=$ latdeg_plus5

else if (latdeg_minus5.1e.60.0_r8.and.Iatdeg_plus5.ge.60.0_r8) then

$\mathrm{de}=(\operatorname{ABS}(60 . r 8-($ latdeg minus5) $) / 10 . r 8) *$ de minus5 \&

$+(\operatorname{ABS}(60 \cdot-r 8-($ latdeg plus 5$)) / 10 \cdot \bar{r} 8) *$ de $\bar{p} l u s 5$

! other case; Now, the slope should be zèro.

else

endif

$\mathrm{de}=($ de_minus $5+$ de_plus5)/2._r8

!Final calculation of de:

!Limits to constrain de to only the observed range and avoid extrapolation.

if (de.1t. 11. r8) then $\mathrm{de}=11 . r 8$

endif

if (de .gt. 106.r8) then

$\mathrm{de}=106 . \mathrm{r} 8$

endif

return

end subroutine deitabhet 